\title{
Compreendendo Comportamentos Emergentes em Sistemas-de-Sistemas por meio de Simulação de Software
}

\author{
Kanan C. Silva ${ }^{1}$, Flávio E. A. Horita ${ }^{1}$ \\ ${ }^{1}$ Centro de Matemática, Computação e Cognição (CMCC) \\ Universidade Federal do ABC (UFABC)
}

Av. dos Estados, 5001 - Bangú - 09210-580 - Santo André - SP - Brasil

\{kanan.silva, flavio.horita\}@ufabc.edu.br

\begin{abstract}
This research is in the context of Systems of Systems (SoS), a class of complex systems composed from other independent systems, which have their own characteristics and functionalities. The interaction among the constituents of a SoS might produce new behaviours, known as emergent behaviours, about which still there is not full dominance regarding modeling and simulation. Thus, this paper intends to collaborate to advance at this understanding by means of the elaboration of a simulator which permits comprehend how the inclusion and/or exclusion of one or more constituents of a SoS affects its global behaviour.
\end{abstract}

Resumo. Esta pesquisa está inserida no contexto de Sistemas de Sistemas (SoS), uma classe de sistemas complexos compostos por outros sistemas independentes, que possuem suas próprias características e funcionalidades. A interação entre os constituintes de um SoS pode produzir comportamentos novos, os chamados comportamentos emergentes, sobre os quais ainda não há pleno domínio à respeito da modelagem e simulação. Assim, este trabalho se propõe a colaborar para o avanço deste entendimento, por meio da elaboração de um simulador que permita entender como a inclusão elou remoção de um ou mais constituintes de um SoS afeta seu comportamento global.

\section{Introdução}

Sistemas-de-Sistemas (SoS) são uma classe de sistemas complexos compostos por outros sistemas individuais (os sistemas constituintes). Originalmente estudados e utilizados no contexto militar dos Estados Unidos na década de 1990, posteriormente eles acabaram ganhando aplicações em áreas como saúde, transporte, energia, gestão de desastres e Internet-das-Coisas [Inocêncio et al. 2019] e, atualmente, estão presentes nos Grandes Desafios da Pesquisa em Sistemas de Informação no Brasil [Boscarioli et al. 2017]. Cada um dos constituintes em um SoS possui sua independência e missões próprias e a integração destes constituintes colabora para o cumprimento de missões globais, as quais nenhum dos sistemas participantes é capaz de realizar sozinho. Segundo Maier [Maier 2014], os SoS podem ser classificados em cinco categorias:

1. Closed SoS: uma entidade gerencia o sistema e controla os objetivos, financiamento e autoridade dos constituintes para se atingir um objetivo;

2. Acknowledged: os objetivos individuais dos constituintes são atendidos em paralelo aos objetivos globais do SoS e a entidade gerenciadora utiliza sua autoridade e recursos para permitir que o SoS trabalhe melhor; 
3. Open SoS: a entidade que gerencia as demais não exerce controle sobre os constituintes, os quais devem ser convencidos a colaborar de maneira voluntária com o SoS, para atendimento do objetivo global;

4. Virtual SoS: não há objetivo global ou entidade gerenciadora, a interação é realizada de maneira deliberada, o que indiretamente proporciona comportamentos emergentes;

5. Organic SoS: são como Virtual SoS, porém, em contextos sociais.

A colaboração entre os componentes de um SoS pode proporcionar novas funcionalidades, os chamados comportamentos emergentes [Maier 2014], os quais podem ser classificados em [Mittal and Rainey 2015]:

1. Simple: podem ser previstos por modelos simplificados do SoS. São produzidos em complexidade inferior em modelos de abstração e apenas comportamentos intencionais previstos irão emergir;

2. Predicted: são reprodutíveis de maneira consistente em simulações do sistema, mas não em modelos estáticos. São parcialmente previstos antecipadamente (comportamentos desejados são previstos, porém indesejados também podem aparecer);

3. Strong: são consistentes com as propriedades conhecidas do $\mathrm{SoS}$, mas não são reprodutíveis em modelo algum do sistema. Simulações podem reproduzir o comportamento de maneira inconsistente e sem prever onde a propriedade irá ocorrer (apesar da existência dos comportamentos desejados, os não-previstos são a maioria);

4. Spooky: Inconsistente com as propriedades conhecidas do SoS, não reprodutíveis ou capazes de serem objetos de simulação.

Neste contexto, este trabalho busca compreender, modelar e simular os comportamentos emergentes em um SoS, avaliando como a inclusão e/ou exclusão de um ou mais constituintes afeta seu comportamento global.

\section{Apresentação do Problema}

Atualmente SoS oferecem suporte a missões em domínios críticos, tais como emergências e gerenciamento de resposta a crises, por esta razão necessitam ser confiáveis e precisos, pois a segurança de pessoas depende de seus serviços. Contudo, um comportamento emergente é incerto e, às vezes, difícil de ser mapeado e modelado.

Cabe ressaltar que tais comportamentos podem contribuir para se atender aos objetivos de negócio por trás do SoS, mas também podem trazer graves consequências, tais como prejuízos financeiros ou ameaças à vida humana, por exemplo, dependendo das circunstâncias. Além disto, os SoS e estes comportamentos possuem propriedades e características específicas, de acordo com seus tipos. Assim, dependendo de qual sistema é removido ou incluído em um SoS, isto pode afetar em maior ou menor grau o comportamento global gerado, além do cumprimento das missões e requisitos do SoS e a entrega de features pelos mesmos. Por exemplo, em um SoS de monitoramento do nível de um rio, a inclusão de um novo sistema constituinte em uma área antes não monitorada pode ampliar a compreensão da situação e, assim, alertar preventivamente no caso da iminência de enchentes. O mesmo acontece no contrário, caso um sistema seja removido, isso poderá 
reduzir a precisão do alerta. Nota-se, portanto, a importância de se identificar e mapear estas variáveis.

Neste contexto, simulações permitem a validação e avaliação de diferentes cenários, além da confiabilidade de um SoS, utilizando ambientes controlados, a fim de se verificar sua resposta, bem como eventuais lacunas que poderiam causar falhas [Neto et al. 2017].

\section{Projeto de Pesquisa}

De acordo com o exposto anteriormente, a presente pesquisa pretende responder à seguinte questão: Como modelar os comportamentos emergentes e compreender seus impactos em um SoS?

Este trabalho pretende verificar a aplicabilidade da teoria dos grafos como uma alternativa para modelar o comportamento emergente em SoS, uma vez que é possível fazer um paralelo entre os constituintes de um SoS e suas relações com os elementos presentes nos princípios desta teoria. Como mostra a Figura 1, nesta proposta de abordagem, cada sistema constituinte será um vértice do grafo, enquanto as conexões entre os sistemas serão representadas pelas arestas, as quais terão seus pesos atribuídos de acordo com a possibilidade de ocorrência destas relações. No exemplo da Figura 1, as linhas cheias representam relações regulares entre os sistemas, enquanto um exemplo de comportamento emergente está denotado pelas linhas pontilhadas. Portanto, a combinação de caminhos no grafo permitirá o surgimento de tais comportamentos.

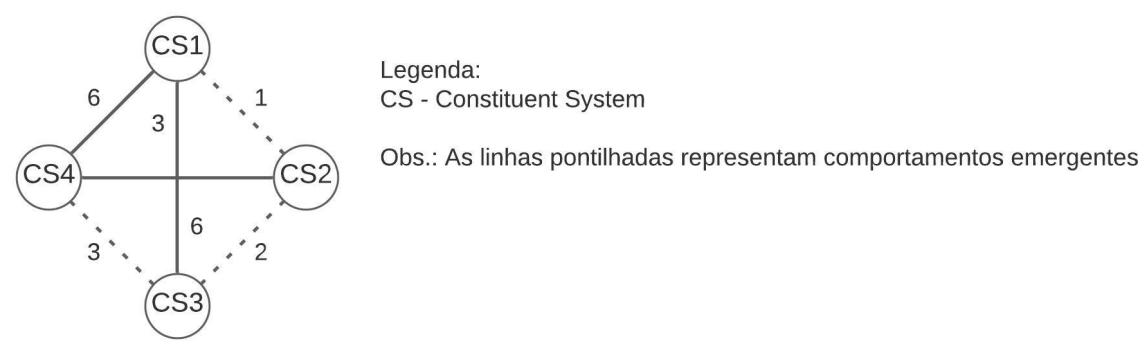

Figura 1. Exemplo de abordagem utilizando Teoria dos Grafos

\section{Projeto de Avaliação}

A avaliação do projeto de pesquisa será realizada em três fases distintas, porém complementares. Primeiramente, será realizado um processo de mapeamento sistemático da literatura, no qual diversos dados serão extraídos e possibilitarão obter uma taxonomia com as características e os fatores influência na gestão dos comportamentos emergentes de um SoS, além de um melhor entendimento sobre o que é possível simular. Em segundo lugar, serão avaliadas as ferramentas e dispositivos existentes para o projeto da futura plataforma de simulação a ser construída. Por fim, um estudo de caso será conduzido em um sistema de gestão de resposta a desastres baseado nas pesquisas de [Horita et al. 2018][Horita et al. 2019][Horita et al. 2020], as quais propõem um framework conceitual com os fatores (dimensões e pilares) que podem otimizar o processo de decisão nas salas de controle de centros destinados ao monitoramento de riscos e alertas precoces. Os dados advindos desta etapa fornecerão subsídios importantes para o 
andamento do projeto, permitindo nortear os próximos passos, dentro dos objetivos específicos estabelecidos, e traçar as estratégias adequadas para se atingir o objetivo geral e responder à pergunta de pesquisa.

\section{Atividades Realizadas}

Está em andamento um mapeamento sistemático da literatura, a fim de se compreender o real estado da arte com respeito à simulação de comportamentos emergentes em SoS. Baseado nas condições e critérios contidos em [Dyba et al. 2007], foram escolhidas referências notáveis na área de Engenharia de Software e Sistemas de Informação, a saber: ACM Digital Library ${ }^{1}$, IEEE Xplore ${ }^{2}$, Wiley Online Library ${ }^{3}$, Springer Link ${ }^{4}$ e ScienceDirect ${ }^{5}$. Foi aplicada a seguinte string de busca:

("Emergent Behaviour"OR "Emergent Behavior") AND ("System of Systems" OR "Systems of Systems" OR "System-of-System" OR "Systems-of-Systems") AND ("Simulation")

Apenas artigos situados entre 2011 e 2021 foram considerados para esta etapa, uma vez que os mesmos representam a maioria dos trabalhos encontrados. Foram obtidos 856 artigos entre as bases escolhidas, dos quais 159 atendem preliminarmente ao critério de inclusão, ou seja, abordam implicita ou explicitamente simulação de comportamentos emergentes em SoS e, portanto, passaram à fase de extração dos dados, etapa atualmente em curso. Posteriormente, será construído um simulador onde serão identificados e analisados tais comportamentos em um estudo de caso.

\section{Considerações Finais}

A compreensão do comportamento emergente em SoS é importante, pois permite aos gestores de tais sistemas atuarem para que os mesmos cumpram seus requisitos e respondam adequadamente conforme suas missões e propósitos, dado o ambiente crítico no qual normalmente se encontram e o nível de confiabilidade e precisão ao qual são exigidos.

Por meio dos resultados deste trabalho, espera-se contribuir para um melhor entendimento das características dos comportamentos emergentes de um $\mathrm{SoS}$, bem como suas aplicações e consequências para o SoS em questão, permitindo aos responsáveis atuarem de maneira a atingir os objetivos do negócio. Entre as contribuições científicas esperadas estão a identificação dos fatores de influência nos comportamentos emergentes de um SoS, a partir do mapeamento sistemático realizado (descritivo), uma metodologia para se modelar tais comportamentos (prescritivo), além das lições aprendidas e recomendações geradas a partir do processo simulação. Como contribuição tecnológica teremos o simulador para modelar e avaliar comportamentos emergentes em um SoS.

\section{Agradecimentos}

Os autores agradecem ao apoio financeiro do Conselho Nacional de Desenvolvimento Científico e Tecnológico (CNPq), Processo Nro 437937/2018-6.

\footnotetext{
${ }^{1}$ https://dl.acm.org/

${ }^{2}$ https://ieeexplore.ieee.org/

${ }^{3}$ https: //onlinelibrary.wiley.com/

${ }^{4}$ https://link.springer.com/

${ }^{5}$ https://wWw. sciencedirect.com/
} 


\section{Referências}

Boscarioli, C., Araujo, R. M., and Maciel, R. S. P. (2017). I GranDSI-BR - Grand Research Challenges in Information Systems in Brazil 2016-2026. Special Committee on Information Systems (CE-SI). Brazilian Computer Society (SBC). ISBN: [978-857669-384-0]. 184p.

Dyba, T., Dingsoyr, T., and Hanssen, G. K. (2007). Applying systematic reviews to diverse study types: An experience report. In First International Symposium on Empirical Software Engineering and Measurement (ESEM 2007), pages 225-234.

Horita, F., Baptista, J., and de Albuquerque, J. P. (2020). Exploring the use of IoT data for heightened situational awareness in centralised monitoring control rooms. Information Systems Frontiers.

Horita, F. E., de Albuquerque, J. P., and Marchezini, V. (2018). Understanding the decision-making process in disaster risk monitoring and early-warning: A case study within a control room in brazil. International Journal of Disaster Risk Reduction, 28:22-31.

Horita, F. E. A., Rhodes, D. H., Inocêncio, T. J., and Gonzales, G. R. (2019). Building a conceptual architecture and stakeholder map of a system-of-systems for disaster monitoring and early-warning. In Proceedings of the XV Brazilian Symposium on Information Systems. ACM.

Inocêncio, T. J., Gonzales, G. R., Cavalcante, E., and Horita, F. E. A. (2019). Emergent behavior in system-of-systems. In Proceedings of the XXXIII Brazilian Symposium on Software Engineering - SBES 2019. ACM Press.

Maier, M. W. (2014). The role of modeling and simulation in system of systems development.

Mittal, S. and Rainey, L. (2015). Harnessing emergence: The control and design of emergent behavior in system of systems engineering. In Proceedings of the Conference on Summer Computer Simulation, SummerSim '15, page 1-10, San Diego, CA, USA. Society for Computer Simulation International.

Neto, V. V. G., Paes, C. E. B., Garcés, L., Guessi, M., Manzano, W., Oquendo, F., and Nakagawa, E. Y. (2017). Stimuli-SoS: a model-based approach to derive stimuli generators for simulations of systems-of-systems software architectures. Journal of the Brazilian Computer Society, 23(1). 